\title{
Study Protocol Version Acronym
}

National Cancer Institute

\section{Source}

National Cancer Institute. Study Protocol Version Acronym. NCI Thesaurus. Code C94108.

The non-unique initials or abbreviated name used for identification of the study. 